\title{
Transformation formulas in quantum cohomology
}

\author{
Prakash Belkale
}

\begin{abstract}
We discuss a natural action of the center of $G$ on the Gromov-Witten numbers of $G / B$ 's and $G / P$ 's. This action is suggested by some problems in representation theory. The quantum Schubert calculus of Grassmannians is an easy consequence of this action. We also strengthen a theorem of Fulton and Woodward, in the case of Grasmannians.
\end{abstract}

\section{Introduction}

It is known [AW98, Bel01] that the problem of determining the conditions on conjugacy classes $\bar{A}_{1}, \ldots, \bar{A}_{s}$ in $S U(n)$, so that these lift to elements $A_{1}, \ldots, A_{s} \in S U(n)$ with $A_{1} A_{2} \ldots A_{s}=1$, is controlled by quantum Schubert calculus of Grassmannians. Teleman and Woodward [TW03] have recently generalized this to an arbitrary simple simply connected compact group $K$. If $G$ is the complex simple subgroup (whose real points are $K$ ), then the role played by the Grassmanians is replaced by the homogeneous spaces $G / P$ for $P$ a maximal parabolic subgroup.

In the case of $S U(n)$ (and similarly for $K$ ), there is a natural 'action' of the center of $S U(n)$ on the representation theory side, namely if $c_{1}, \ldots, c_{s}$ are central elements with $c_{1} c_{2} \ldots c_{s}=1$, then these act on the set of conjugacy classes $\bar{A}_{1}, \ldots, \bar{A}_{s}$ in $S U(n)$ liftable to elements $A_{1}, \ldots, A_{s} \in S U(n)$ with $A_{1} A_{2} \ldots A_{s}=1$, the action being just multiplying $\bar{A}_{i}$ by $c_{i}$. This action is well defined on the level of conjugacy classes because the $c_{i}$ are central.

This suggests a natural transformation property of Gromov-Witten numbers of the Grassmannians under the action of the center. This property was proved in [AW98] as a consequence of the known description of quantum Schubert calculus [Ber97]. Postnikov proved a similar property for the complete flag manifold $S L_{n} / B$ [Pos00]. Our aim initially was to make clear that the numbers coincide because if suitably interpreted they count points in the 'same intersection'.

The aim of this article is twofold. The first aim is to prove the transformation formulas geometrically and in complete generality (for any simple simply connected complex Lie group). The second is to show that these formulas determine quantum Schubert calculus in the case of Grassmannians (Bertram's Schubert calculus). We also give a strengthening in the case of Grassmannians of a theorem of Fulton and Woodward on the lowest power of $q$ appearing in a (quantum) product of Schubert classes in $G / P$, where $P$ is a maximal parabolic subgroup.

Let us now describe these transformation formulas; see $\S 1$ for the notation. Let $G$ be a simple simply connected complex algebraic group. We first construct a map $\phi: C \rightarrow W$, where $C$ is the center of $G$ and $W$ the Weyl group. Let $Z$ be a homology class of $G / P$, where $P$ is an arbitrary parabolic subgroup (not necessarily maximal). Let $c_{1}, \ldots, c_{s}$ be central elements with product equal to one. Let $w_{1}, \ldots, w_{s}$ be elements of a suitable right quotient of $W$, then the transformation formulas take the shape

$$
\left\langle X_{\phi\left(c_{1}\right) w_{1}}, \ldots, X_{\phi\left(c_{s}\right) w_{s}}\right\rangle_{Z^{\prime}}=\left\langle X_{w_{1}}, \ldots, X_{w_{s}}\right\rangle_{Z},
$$

where $Z^{\prime}$ is a homology class determined by $Z$ and the rest of the data.

Received 2 May 2002, accepted in final form 2 April 2003.

2000 Mathematics Subject Classification 14N15 (primary), 14N35 (secondary).

Keywords: Grassmann variety, cohomology, parabolic subgroup.

This journal is (C) Foundation Compositio Mathematica 2004. 
It happens that in some cases, $Z^{\prime}$ is a simpler homology class than $Z$; for instance, $Z^{\prime}$ could be zero when $Z$ is not. This allows for the reduction of the quantum terms to the classical ones. This program works in the (ordinary) Grassmannian case.

There exist simple simply connected groups with trivial center. In this case, the transformation formulas do not give any information. There may be an extension of these transformation formulas to the non-miniscule case. Such an extension is not apparent from the representation theory side. The transformation formulas give some information about quantum cohomology for $G / P$ if $G$ has a center.

One final comment is that even in the classical case of cohomology, the transformation formulas give vanishing statements. For example, if $Z^{\prime}$ turns out to be negative and $Z=0$, then we get a vanishing statement of certain intersection numbers.

Many of the results in this paper are new proofs of older results using methods which seem both natural and elementary (to the author). It is perhaps worth pointing out what is essentially new in this paper: the transformation formulas in the usual partial flag manifold case (that is $S L(n, \mathbb{C}) / P$ where $P \neq B$ is a parabolic subgroup which is not maximal); the exact determination of the lowest order terms in the quantum product of two Schubert cycles in (usual) Grassmannians; and the natural extension of the transformation formulas to all groups.

There is recent literature on the quantum cohomology of $G / P$ that should be mentioned: Kim [Kim99] worked out the quantum cohomology of $G / B$ (with generators and relations). Kresch and Tamvakis computed the quantum Schubert calculus of the Lagrangian and Orthogonal Grasmannians [KT03, KT04]. There are also recent preprints by Mare [Mar02] on Schubert calculus for the $G / B$ case and by Woodward [Woo02] on reducing the general $G / P$ case to the $G / B$ case. So, computationally we seem to be closing in on a complete picture.

For $G / P$ there is already considerable information coming from usual cohomology and combined with the transformation formulas (in some cases), one can hope that sufficiently many of the quantum terms become classical (as is the case for Grassmannians). However, in practice, computation of the entire quantum cohomology involves some vanishing statements too. For instance, to prove that the Pieri formulas for Lagrangian Grassmannian do not involve $q^{2}$ terms (which we managed for the ordinary Grassmannian case) seems to require additional reasoning. Kresch and Tamvakis achieve this by studying the $\tilde{Q}$ polynomials of Pragacz and Ratajski.

Buch [Buc03] has recently given new proofs of Bertram's quantum Schubert calculus, using very different methods.

\section{Some representation theory}

\subsection{Notation}

We review some basic representation theory in this section. For proofs refer to Bourbaki [Bou02].

Let $G$ be a simple simply connected complex algebraic group. Let $\mathfrak{g}$ be its Lie algebra. Let $B$ be a Borel subgroup, $T \subset B$ a maximal torus and let

$$
\mathfrak{g}=\mathfrak{h} \bigoplus_{\alpha} \mathfrak{g}_{\alpha},
$$

where the $\alpha$ 's belong to the subset of roots $R$ in $\mathfrak{h}^{*}$. The set $R$ is partitioned into the set of positive roots $R^{+}$and negative roots $R^{-}$, and the Lie algebra of $B$ is

$$
\mathfrak{b}=\mathfrak{h} \bigoplus_{\alpha} \mathfrak{g}_{\alpha}
$$

with the $\alpha$ 's in $R^{+}$. Also, define $\Delta$ to be the set of simple roots. The Weyl group $W$ is defined to 


\section{P. Belkale}

be $N(T) / T$, where $N(T)$ is the normalizer of $T$ which acts on $\mathfrak{h}$ and $\mathfrak{h}^{*}$. If $\alpha$ is a root, we have elements $w_{\alpha} \in W, H_{\alpha} \in \mathfrak{h}$, so that $w_{\alpha}$ acts on $\mathfrak{h}^{*}$ by

$$
w_{\alpha}(\beta)=\beta-\beta\left(H_{\alpha}\right) \alpha
$$

and this map preserves the roots, is a reflection and takes $\alpha$ to $-\alpha$. The real vector space spanned by $H_{\alpha}$ is denoted by $\mathfrak{h}_{\mathbb{R}}$.

The action of $w_{\alpha}$ on $\mathfrak{h}$ is given by

$$
w_{\alpha}(H)=H-\alpha(H) H_{\alpha} .
$$

It is also known that $w_{\alpha}$ 's generate $W$. The affine Weyl group $W_{\text {aff }}$ is defined to be the set of automorphisms of $\mathfrak{h}$ generated by $W$ and translations by $H_{\alpha}$ for $\alpha \in R$.

\subsection{Conjugacy classes}

Let $K$ be the maximal (connected) compact subgroup of $G$ associated to the root data. If $\mathfrak{k}$ is the Lie algebra of $K$, then $\mathfrak{k} \bigotimes_{\mathbb{R}} \mathbb{C}=\mathfrak{g}$. Let $T_{K}=T \cap K$ be the maximal torus in $K$, with Lie algebra $i \mathfrak{h}_{\mathbb{R}}$. The following are standard facts.

1) $T_{K} \rightarrow K$ induces a surjection on conjugacy classes.

2) $x_{1}, x_{2} \in T_{K}$ are conjugate in $K$ if and only if there exists $w \in W$ with $\operatorname{Ad}(w) x_{1}=x_{2}$.

3) Let Exp $: \mathfrak{h}_{K} \rightarrow T_{K}$ be the exponential map from the Lie algebra of $T_{K}$ to $T_{K}$. The kernel of this map is $\Gamma(T)=\mathbb{Z}$-span $\left\{2 \pi i H_{\alpha} \mid \alpha \in R\right\}$. This follows from the simply connectedness of $G$.

4) If $t_{1}, t_{2} \in \mathfrak{h}_{K}$, then $\operatorname{Exp}\left(t_{1}\right)$ and $\operatorname{Exp}\left(t_{2}\right)$ are conjugate in $K$ if and only if there exists $w \in W$ with

$$
w\left(t_{1}\right)-t_{2} \in \Gamma(T)
$$

Putting this all together, we find that the map $\mathfrak{h}_{\mathbb{R}} \rightarrow T_{K}$ given by $t \rightarrow \operatorname{Exp}(2 \pi i t)$ induces an isomorphism $\mathfrak{h}_{\mathbb{R}} / W_{\text {aff }} \rightarrow$ conjugacy classes in $K$.

\subsection{Fundamental chamber and the center}

Let $L_{\alpha, k}=\left\{x \in \mathfrak{h}_{\mathbb{R}} \mid \alpha(x)=k\right\}$. The affine Weyl group is then the group generated by reflections in $L_{\alpha, k}$ for $k \in \mathbb{Z}$. Finally, let $\tilde{\alpha}$ be the highest weight for the adjoint representation.

Theorem 1 (Fundamental chamber for affine Weyl group). Let $C=\left\{x \in \mathfrak{h}_{\mathbb{R}} \mid \alpha(x)>0\right.$ for $\left.\alpha \in R^{+}, \tilde{\alpha}(x)<1\right\}$.

1) $C$ is a connected component of $\mathfrak{h}_{\mathbb{R}}-\bigcup_{\alpha \in R, k \in \mathbb{Z}} L_{\alpha, k}$.

2) If $C^{\prime}$ is any other component, there is a unique $w \in W_{\text {aff }}$ with $w(C)=C^{\prime}$.

3) Let $\bar{C}$ be the closure of $C$, then the composite $p: \bar{C} \rightarrow \mathfrak{h}_{\mathbb{R}} / W_{\text {aff }} \rightarrow$ conjugacy classes in $K$ is a homeomorphism.

We now give the description of the center. For this, let $S=\{x \in \bar{C} \mid \alpha(x) \in \mathbb{Z}$, for all $\alpha \in R\}$. Finally, write $\tilde{\alpha}=\sum_{\alpha \in \Delta} n_{\alpha} \alpha$.

THEOREM 2.

1) The map $p: \bar{C} \rightarrow$ conjugacy classes in $G$, takes $S$ to center $(K)$.

2) Define $x_{\alpha}$ for $\alpha \in \Delta$ by the formula $\beta\left(x_{\alpha}\right)=\delta_{\alpha, \beta}$ for $\alpha, \beta \in \Delta$. Then, $S=\{0\} \cup\left\{x_{\alpha} \mid \alpha \in\right.$ $\left.\Delta, n_{\alpha}=1\right\}$.

Definition 1. For $c \in \operatorname{center}(K)$, define $h_{c}=p^{-1}(c)$. Note that if $c \neq 1, h_{c}=x_{\alpha}$ for some $\alpha \in \Delta$ and $h_{c}=0$ if $c=1$. 


\subsection{A map from the center to the Weyl group}

Let $c \in \operatorname{center}(G)(=\operatorname{center}(K))$. Consider the set $C^{\prime}=C-h_{c}\left(\right.$ where $h_{c}$ was defined in Definition 1$)$. It is easy to see that this is a connected component of $\mathfrak{h}_{\mathbb{R}}-\bigcup_{\alpha \in R, k \in \mathbb{Z}} L_{\alpha, k}$. Therefore, we have

$$
C-h_{c}=w_{c}^{-1}(C)+t,
$$

where $w_{c} \in W$ and $t \in \mathbb{Z}$-span of $H_{\beta}, \beta \in R$.

For $x \in \mathfrak{h}_{\mathbb{R}}$, define $y_{c}(x)=w_{c}\left(x-h_{c}-t\right)$. Then we have the equation

$$
x-h_{c}=w_{c}^{-1}\left(y_{c}(x)\right)+t
$$

and also that $x \in C \Rightarrow y_{c}(x) \in C$. Hence, $x \in \bar{C} \Rightarrow y_{c}(x) \in \bar{C}$.

Now put $x=h_{c}$. We get $w_{c}^{-1}\left(y_{c}(x)\right)=-t$, this gives $y_{c}(x)$ is zero in $\mathfrak{h}_{\mathbb{R}} / W_{\text {aff }}$ and, by Theorem 1 , we get $y_{c}(x)=0$ so $t=0$.

We therefore have the following.

Lemma 1 (Map from the center to the Weyl group). For $c$ in center $(K)$, let $h_{c}=p^{-1}(c)$. Then there exists a $w_{c} \in W$ so that the equation $C-h_{c}=w_{c}^{-1}(C)$ holds. Furthermore, the map center $(G) \rightarrow W$ is an injective homomorphism of groups.

Proof. The only part not proved yet is that $c \mapsto w_{c}$ is a homomorphism of groups. For this, let $c_{1}, c_{2}$ be central elements. Let $h_{c_{1}}, h_{c_{2}}, h_{c_{1} c_{2}}$ correspond to $c_{1}, c_{2}$ and $c_{1} c_{2}$, respectively (where $h_{c}=p^{-1}(c)$ as before).

It is clear that $h_{c_{1}}+h_{c_{2}}=h_{c_{1} c_{2}}+t$ with $t \in \mathbb{Z}$-span of $H_{\delta}, \delta \in R$. hence

$$
\begin{aligned}
C-h_{c_{1} c_{2}} & =C-h_{c_{1}}-h_{c_{2}}+t \\
& =w_{c_{1}}^{-1}\left(C-h_{c_{2}}\right)+\left(w_{c_{1}}^{-1}\left(h_{c_{2}}\right)-h_{c_{2}}\right)+t \\
& =w_{c_{1}}^{-1} w_{c_{2}}^{-1}(C)+t_{1}+t,
\end{aligned}
$$

where $t_{1}$ and $t$ are in $\mathbb{Z}$-span of $H_{\delta}, \delta \in R$. The proof is therefore complete.

We can describe the element $w_{c}$ more concretely; for this, first note the following.

1) If $x \in C$ then $y_{c}(x) \in C$.

2) For $\beta \in R, \beta \in R^{+}$if and only if $\beta(x)>0$ for any $x \in C$.

3) $w_{c}(\beta)\left(y_{c}(x)\right)=\beta\left(w_{c}^{-1}\left(y_{c}(x)\right)\right)=\beta(x)-\beta\left(h_{c}\right)$.

We therefore have the following description of $w_{c}$.

Lemma 2. In the situation above and with $\beta \in R^{+}$:

1) if $\beta\left(h_{c}\right)=0$, then $w_{c}(\beta) \in R^{+}$;

2) if $\beta\left(h_{c}\right)=1$, then $w_{c}(\beta) \in R^{-}$.

Remark 1. From a computational point of view the above lemma determines $w_{c}$ completely. We could have taken this as a definition, but then we would have had to connect it to the fundamental chamber.

\subsection{Parabolics associated to central elements}

Fix $c_{1}, c_{2}$ belonging to center $(G)$ with $c_{1} c_{2}=1, c_{1} \neq 1, c_{2} \neq 1$. Let $x_{\alpha}, x_{\beta}$ be their representatives in $\bar{C}$. 


\section{P. Belkale}

Define parabolic subgroups $P_{1}, P_{2} \supset B$ by defining their Lie algebras

$$
\begin{aligned}
& \mathfrak{p}_{1}=\mathfrak{h} \bigoplus_{\gamma \mid \gamma\left(x_{\alpha}\right) \geqslant 0} \mathfrak{g}_{\gamma} \\
& \mathfrak{p}_{2}=\mathfrak{h} \bigoplus_{\gamma \mid \gamma\left(x_{\beta}\right) \geqslant 0} \mathfrak{g}_{\gamma}
\end{aligned}
$$

and their Levi subgroups $Q_{1}, Q_{2}$ by defining their Lie algebras

$$
\begin{gathered}
\mathfrak{q}_{1}=\mathfrak{h} \bigoplus_{\gamma \mid \gamma\left(x_{\alpha}\right)=0} \mathfrak{g}_{\gamma} \\
\mathfrak{q}_{2}=\mathfrak{h} \bigoplus_{\gamma \mid \gamma\left(x_{\beta}\right)=0} \mathfrak{g}_{\gamma} .
\end{gathered}
$$

The fact that these are closed subgroups follows from the P's being standard parabolics and $Q$ 's being the centralizers of $x_{\alpha}, x_{\beta}$.

These are related due to the relation $c_{1} c_{2}=1$.

Lemma 3. $Q_{2}=\operatorname{Ad}\left(w_{c_{1}}\right)\left(Q_{1}\right)$ or $\gamma\left(x_{\alpha}\right)=0$ if and only if $\left(w_{c_{1}}(\gamma)\right)\left(x_{\beta}\right)=0$.

Proof. This follows from $-x_{\alpha}=w_{c_{1}}^{-1}\left(x_{\beta}\right)$.

Lemma 4. $\left(\operatorname{Ad}\left(w_{c_{1}}\right)\left(P_{1}\right)\right) \cap P_{2}=Q_{2}$ and this is a transverse intersection.

Proof. The first statement follows from

$$
\left(w_{c_{1}}(\gamma)\right)\left(x_{\beta}\right)=-\gamma\left(x_{\alpha}\right)
$$

The transversality statement follows from the same equation (counting dimensions).

Corollary 1. If $g_{1}, g_{2}$ are general elements of $G$, the set $\left(w_{c_{1}} P_{1} g_{1}\right) \cap\left(P_{2} g_{2}\right)$ is non-empty.

Proof. If $g_{1}=w_{c_{1}}^{-1}, g_{2}=1$, then this follows from the above lemma. Then apply standard intersection theory (local).

\section{Algebraic geometry preliminaries on $G / P$}

\subsection{Line bundles on $G / P$ and $G / B$}

Let $P$ be a parabolic containing $B$. We then have a natural surjection $G / B \rightarrow G / P$. It is known that this induces injections on the Picard groups. Our goal here is to recall the standard facts on describing all the line bundles on $G / B$ and those that descend to $G / P$.

Let $W L=$ weight lattice of $\mathfrak{g}$. This is the subset of $\mathfrak{h}^{*}$ spanned by elements $\omega$ so that $\omega\left(H_{\alpha}\right) \in \mathbb{Z}$ for all $\alpha \in R$. It has a $\mathbb{Z}$-basis $\left\{\omega_{\alpha} \mid \alpha \in \Delta\right\}$, where

$$
\omega_{\alpha}\left(H_{\beta}\right)=\delta_{\alpha, \beta},
$$

for all $\alpha, \beta \in \Delta$.

There is a natural isomorphism $\psi: W L \rightarrow \operatorname{Pic}(G / B)$. The map is defined as follows: for each $\omega \in W L^{+}$there exists a representation $\rho: G \rightarrow G L(V)$ with highest weight $\omega$. Let the highest weight vector be $v \in V$. Then there is a map $G / B \rightarrow \operatorname{Orb}(\mathbb{C} v) \subset \mathbb{P}(V)$, where $\operatorname{Orb}(\mathbb{C} v)$ is the orbit of the line $\mathbb{C} v$. The map then takes $\omega$ to pull back of $\mathcal{O}(1)$ by the map above.

The subset $W L_{P}$ of weights that descend to line bundles on $G / P$ are just those elements $\omega$ which satisfy if $\mathfrak{g}_{\alpha} \bigoplus \mathfrak{g}_{-\alpha} \subset \mathfrak{p}$, then $\omega\left(H_{\alpha}\right)=0$. 
The second homology group $H_{2}(G / P, \mathbb{Z})$ can be naturally considered as the dual Hom $\left(W L_{P}, \mathbb{Z}\right)$ by Poincare duality. Note that the homology class of $f_{*}([C])$, where $f: \mathbb{P}^{1} \rightarrow G / P$ corresponds to the map $W L_{P} \rightarrow \mathbb{Z}$ obtained by taking the 'degree of the pullback bundle'.

Next we describe the first Chern classes of the tangent bundles of $G / B$ and $G / P$.

1) First Chern class of $T_{G / B}$ : this is $\psi\left(\sum_{\alpha \in R^{+}} \alpha\right)$.

2) First Chern class of $T_{G / P}$ : this is $\psi\left(\sum_{\alpha \in R, \mathfrak{g}_{\alpha} \subset \mathfrak{p}} \alpha\right)$.

\subsection{Cell decomposition and cohomology of $G / P$}

Let $P \supset B$ be a parabolic subgroup. Let $R_{P}$ be the set of roots $\alpha$ such that $\mathfrak{g}_{\alpha} \bigoplus \mathfrak{g}_{-\alpha} \subset \mathfrak{p}$. Let $\Delta_{P}=R_{P} \cap \Delta$. Finally let $W_{P}$ be the subgroup of the Weyl group generated by the reflections corresponding to elements of $\Delta_{P}$ (or of $R_{P}$ ).

Theorem 3 (Bruhat decomposition). $G / P$ is a disjoint union of the sets $\Lambda_{w}$ for $w \in W / W_{P}$, where $\Lambda_{w}$ is defined to be $B w P \subset G / P$. Let $X_{w}$ be the closure of $\Lambda_{w}$. The codimension of $X_{w}$ is the cardinality of the set

$$
\left|\left\{\alpha \in R \mid \mathfrak{g}_{\alpha} \not \subset \mathfrak{p}, w(\alpha) \notin R^{+}\right\}\right| .
$$

The proof is standard and is by examining the tangent space of $w^{-1} \Lambda_{w}$ at $e \in G / P$.

It is known that the subvarieties $X_{w}$ generate the cohomology (additively) of $G / P$. Finally, recall the definition of 'relative position' $\left[g_{1}, g_{2}\right]$ of two elements $g_{1}, g_{2} \in G$. We define $w=\left[g_{1}, g_{2}\right]$ to be the unique element of the Weyl group so that there exist $b_{1}, b_{2} \in B$ so that $g_{1}=g_{2} b_{1} w b_{2}$ (Bruhat decomposition).

Note the following three properties.

1) $\left[g_{1}, g_{2}\right]=\left[g g_{1}, g g_{2}\right]$ for $g_{1}, g_{2} \in G$.

2) $\left[g_{1} b, g_{2}\right]=\left[g_{1}, g_{2} b\right]=\left[g_{1}, g_{2}\right]$ for $b \in B$.

3) $h \in g \Lambda_{w}$ if and only if $[h, g]=w$.

Analogous definition of relative position can be made of $\left[g_{1}, g_{2}\right]$ where this takes values in $W / W_{P}, g_{1} \in G / P$ and $g_{2} \in G$.

We need one final lemma which relates the codimensions of $X_{w}$ and $X_{w_{c} w}$, where $w_{c}$ is an element of the Weyl group constructed out of a central element $c$ as in the previous section.

Lemma 5. Let $c \in \operatorname{center}(G), w \in W$ with representative $x_{\alpha} \in \bar{C}$. Then $\operatorname{codim}\left(X_{w_{c} w}\right)-\operatorname{codim}\left(X_{w}\right)$ is equal to

$$
\sum_{\beta \in R \backslash R_{P}} w \beta\left(x_{\alpha}\right)
$$

Proof. The quantity we are interested in is

$$
\left|\left\{\alpha \in R \backslash R_{P} \mid w(\alpha) \in R^{+}\right\}\right|-\left|\left\{\alpha \in R \backslash R_{P} \mid\left(w_{c} w\right)(\alpha) \in R^{+}\right\}\right| .
$$

To evaluate the second quantity (using Lemma 2), divide into two cases namely

$$
\left|\left\{\alpha \in R \backslash R_{P} \mid w(\alpha) \in R^{+}, w \beta\left(x_{\alpha}\right)=0\right\}\right|
$$

and

$$
\left|\left\{\alpha \in R \backslash R_{P} \mid w(\alpha) \in R^{-}, w \beta\left(x_{\alpha}\right)=-1\right\}\right| .
$$

Therefore, the quantity we are interested in becomes

$$
\left|\left\{\alpha \in R \backslash R_{P} \mid w(\alpha) \in R^{+}, w \beta\left(x_{\alpha}\right)=1\right\}\right|-\left|\left\{\alpha \in R \backslash R_{P} \mid w(\alpha) \in R^{-}, w \beta\left(x_{\alpha}\right)=-1\right\}\right|
$$




\section{P. Belkale}

and that is what is displayed in the statement of the lemma. Note that our hypotheses imply that if $\beta \in R$, then $\beta\left(x_{\alpha}\right)$ is in the set $\{-1,0,1\}$.

\subsection{Space of maps and Gromov-Witten invariants}

For $X \in H_{2}(G / P, \mathbb{Z})$, let $M_{X}=$ space of maps $f: \mathbb{P}^{1} \rightarrow G / P$ so that $f_{*}\left(\left[\mathbb{P}^{1}\right]\right)=X$. It is known that $M_{X}$ can be given the structure of a smooth quasi-projective variety of dimension $d_{X}$, where $d_{X}=c_{1}\left(T_{G / P}\right) \cap X+\operatorname{dim}(G / P)$.

Of central interest to us in this paper are Gromov-Witten invariants. Recall that we have fixed three points $p_{1}, p_{2}, p_{3}$ on $\mathbb{P}^{1}$ (which we usually take to be $0, \infty, 1$ ).

Definition 2. Let $Z \in H_{2}(G / P, \mathbb{Z}), w_{1}, w_{2}, w_{3} \in W$. Then,

$$
\left\langle X_{w_{1}}, X_{w_{2}}, X_{w_{3}}\right\rangle_{Z}
$$

is defined to be the number of maps (zero if infinite) $f \in M_{Z}$ so that $f\left(p_{i}\right) \in g_{i} X_{w_{i}}, i=1,2,3$ where $g_{i}$ are 'general' points of $G$.

Note that the invariant above is zero unless

$$
\sum \operatorname{codim}\left(X_{w_{i}}\right)=c_{1}\left(T_{G / P}\right) \cap Z+\operatorname{dim}(G / P) .
$$

\section{The transformation formula}

Let $c_{1}, c_{2} \in \operatorname{center}(G)$ with $c_{1} c_{2}=1$. Use the notation of $\S 1.5$ associated with these elements. Let $x_{1}=h_{c_{1}}, x_{2}=h_{c_{2}}$ and $w_{1}=w_{c_{1}}, w_{2}=w_{c_{2}}$.

Theorem 4. Let $Z \in H_{2}(G / P, \mathbb{Z}), u_{1}, u_{2}, u_{3} \in W$, then

$$
\left\langle X_{u_{1}}, X_{u_{2}}, X_{u_{3}}\right\rangle_{Z}=\left\langle X_{w_{1} u_{1}}, X_{w_{2} u_{2}}, X_{u_{3}}\right\rangle_{Z^{\prime}}
$$

where $Z^{\prime}$ as an element of $\operatorname{Hom}\left(W L_{P}, \mathbb{Z}\right)$ is given by

$$
Z^{\prime}(\gamma)=Z(\gamma)-\gamma\left(u_{1}^{-1} x_{1}-x_{1}\right)-\gamma\left(u_{2}^{-1} x_{2}-w_{2} x_{2}\right)
$$

We first check that the codimension condition

$$
\sum \operatorname{codim}\left(X_{u_{i}}\right)=c_{1}\left(T_{G / P}\right) \cap Z+\operatorname{dim}(G / P),
$$

for the left-hand side is the same as that for the right-hand side.

Recall that if $c \in \operatorname{center}(G), w \in W$ with representative $x_{\alpha} \in \bar{C}$, then $\operatorname{codim}\left(X_{w_{c} w}\right)-\operatorname{codim}\left(X_{w}\right)$ is equal to

$$
\sum_{\beta \mid \mathfrak{g}_{\beta} \not \subset p} w \beta\left(x_{\alpha}\right) .
$$

So we have to verify that

$$
\sum_{\beta \mid \mathfrak{g}_{\beta} \not \subset p}\left(u_{1} \beta\left(x_{1}\right)+u_{2} \beta\left(x_{2}\right)\right)
$$

equals

$$
\gamma\left(Z^{\prime}\right)-\gamma(Z)
$$

where

$$
\gamma=-\sum_{\beta \mid \mathfrak{g}_{\beta} \not \subset p} \beta,
$$

which has been proved in Lemma 5 . 
Now fix $g_{1}, g_{2}, g_{3} \in G$, 'elements in a general position'. Pick an element $k \in\left(P_{1} g_{1}^{-1}\right) \cap\left(w_{2} P_{2} g_{2}^{-1}\right)$. There exists such a $k$ because of Corollary 1.

Consider the map $\phi: G / P \rightarrow G / P$ given by left multiplication by $k$. Let $f \in M_{Z}$, we claim $f\left(p_{i}\right) \in g_{i} X_{w_{i}}, i=1,2,3$, if and only if by setting $g=\phi f$, we have $g\left(p_{i}\right) \in k g_{i} X_{w_{i}}, i=1,2,3$. This claim is obvious but we also have $k\left(g_{1}\right) \in P_{1}$ and $k g_{2} \in \operatorname{Ad}\left(w_{c_{2}}\right) P_{2}$. So we might as well assume $g_{1} \in P_{1}$ and $g_{2} \in w_{c_{2}} P_{2}$.

Now suppose $f: \mathbb{P}^{1} \rightarrow G / P$. Let $s$ be the map $\mathbb{P}^{1} \rightarrow G / P$ given by

$$
s(z)=z^{x_{1}} f(z)
$$

where for $t \in T, z \in \mathbb{C}, z^{t}=\operatorname{Exp}(\ln (z) t)$ (a multivalued map). Note that the indeterminacy of $s$ is always central, so as a map to $G / P$ it is well defined on the complement of $\{0,1, \infty\}$. We can extend this to all of $\mathbb{P}^{1}$, because all the functions involved are of bounded growth.

We have to study the effect on the degrees and also on the 'positions' of $s(0), s(1), s(\infty)$.

\subsection{Position of $s(0)$}

We have assumed that $g_{1}$ is in $P_{1}$ and $P_{1}=Q_{1} B$. So let $g_{1}=q_{1} b$. We claim that the element $g_{1}^{\prime}=q_{1} w_{1}^{-1}$ is well defined in $G / P$ (independent of choices). We need that if $q_{1}^{\prime}=q_{1} b$, then $q_{1} w_{1}^{-1}$ and $q_{1} b w_{1}^{-1}$ give the same point in $G / P$. That is, $w_{1} b w_{1}^{-1} \in B$ if $b \in Q_{1} \cap B$. However, this is clear from Lemma 2.

Claim 1. $\left[s(0), g_{1}^{\prime}\right]=w_{1}\left[f(0), g_{1}\right]$.

That is, $\left[s(0), q_{1} w_{1}^{-1}\right]=w_{1}^{-1}\left[f(0), q_{1}\right]$. Let $f(0)=q_{1} b_{1} w b_{2}$ and $f=n(z) f(0)$ where $n(0)=1$. We therefore need to compare

$$
\left[\lim _{z \rightarrow 0} z^{x_{1}} n(z) f(0), g_{1}^{\prime}\right] \quad \text { with }\left[f(0), g_{1}\right] .
$$

Or, if we set $h=q_{1}^{-1} f(0)$, we want to relate

$$
\left[\lim _{z \rightarrow 0}\left(\operatorname{Ad}\left(q_{1}^{-1}\right) z^{x_{1}}\right)\left(\operatorname{Ad}\left(q_{1}^{-1}\right) n(z)\right) h, w^{-1}\right] \quad \text { to }[h, 1] .
$$

It is easy to see that $\operatorname{Ad}\left(q_{1}^{-1}\right) z^{x_{1}}=z^{x_{1}}$. Setting $r(z)=\operatorname{Ad}\left(q_{1}^{-1}\right) n(z)$, we then want to relate

$$
\left[\lim _{z \rightarrow 0} z^{x_{1}} r(z) h, w^{-1}\right] \text { to }[h, 1],
$$

where $r(0)=1$. For this we need the following (and this proves the claim).

Lemma 6. If $d(z)$ is a holomorphic map to $G$, with $d(0) \in B$ then

$$
k=\lim _{z \rightarrow 0} \operatorname{Ad}\left(z^{x_{1}}\right) d(z)
$$

exists with $w_{1} k w_{1}^{-1} \in B$.

Proof. $G$ is generated by the one parameter groups $G_{\alpha}$ for $\alpha \in R$ and $T$. These groups are isomorphic to $\mathbb{C}$ and with an action (Ad) of the torus with $\operatorname{Ad}(t) u=\alpha(t) u$.

If $d(z) \in G_{\alpha}=\mathbb{C}$ given by $d(z)=z^{m}$ then $\operatorname{Ad}\left(z^{x_{1}}\right) d(z)=z^{\alpha\left(x_{1}\right)} d(z)$. Hence, to verify the lemma we need the following.

1) If $\alpha \in R^{+}$then $\alpha\left(x_{1}\right)=0$ implies $w_{1}(\alpha)$ is a positive root, which is known. If $\alpha\left(x_{1}\right)=1$, then $k=1$.

2) If $\alpha \in R^{-}$with $\alpha\left(x_{1}\right)=0$, then clearly $k=1$.

3) If $\alpha \in R^{-}$with $\alpha\left(x_{1}\right)=-1$, then clearly $k$ exists and $w_{1}(\alpha)$ is positive. 


\section{P. Belkale}

\subsection{Position of $s(\infty), s(1)$}

Note that we have chosen $p_{2}=\infty$ in order to simplify the notation.

We have $g_{2} \in w_{2} P_{2}$. Consider the map $\psi: G / P \rightarrow G / P$ by left multiplication by $w_{2}^{-1}$. Write $g_{2}=$ $w_{2} q_{2} b$ for $q_{2} \in Q_{2}$ and $b \in B$. Let $g_{2}^{\prime}=w_{2} q_{2} w_{2}^{-1} b$ as before the $G / P$ class of $g_{2}^{\prime}$ is well-defined. It is then easy to see that $w_{2}\left[f(\infty), g_{2}\right]=\left[s(\infty), g_{2}^{\prime}\right]$. For this it is enough to note that $z^{x_{1}}=(1 / z)^{w_{2} x_{2}}$.

Finally, let $g_{3}^{\prime}=g_{3}$. It is clear that $\left[s(1), g_{3}^{\prime}\right]=\left[f(1), g_{3}\right]$. Next we have to compute the homology class $s_{*}\left(\left[\mathbb{P}^{1}\right]\right)$.

Lemma 7 . If $Z$ is the element in $\operatorname{Hom}\left(W L_{P}, \mathbb{Z}\right)$ corresponding to $f$, then the element $Z^{\prime}$ corresponding to $s$ is

$$
\begin{aligned}
Z^{\prime}(\gamma) & =Z(\gamma)-\gamma\left(u_{1}^{-1} x_{1}-x_{1}\right)-\gamma\left(u_{2}^{-1} x_{2}-w_{2} x_{2}\right) \\
& =Z(\gamma)-u_{1} \gamma\left(x_{1}\right)-u_{2} \gamma\left(x_{2}\right)
\end{aligned}
$$

Proof. It is enough to prove this in the case $\gamma$ positive and integral. Let $L$ be the line bundle on $G / P$ corresponding to $\gamma$. We construct the line bundle corresponding to $\gamma$ in a different (equivalent) manner first. First extend $\gamma$ to a map $\Gamma: P \rightarrow \mathbb{C}^{*}$. Then construct the total space of $L$ as $G \times \mathbb{C} / R$, where $R$ is the equivalence relation $(g, v)=(g p, \Gamma(p) v)$ for $p \in P$. The maps $f, s$ give two line bundles $L_{f}=f^{*}(L), L_{s}=s^{*}(L)$. At a point other than $0, \infty$, construct the map $\psi: L_{f} \rightarrow L_{s}$, by $(\breve{f}(z), 1)$ to $\left(z^{x_{1}} \breve{f}(z), \Gamma\left(z^{x_{1}}\right)\right)$ where $\breve{f}(z)$ is a local lifting of $f$ to a map $\rightarrow G$, and where the same determination of $z^{x_{1}}$ is used in both $z^{x_{1}} \breve{f}(z)$ and in $\Gamma\left(z^{x_{1}}\right)$.

It is immediate to see that $\psi$ is an isomorphism of bundles outside of $\{0, \infty\}$. Let us analyze this map first at $z=0$. Lift $f$ to a map $\breve{f}$ to $G$. Then $(\breve{f}, 1)$ is a local section of $f$ and this is mapped by $\psi$ to $\left(z^{x_{1}} \breve{f}, \Gamma\left(z^{x_{1}}\right)\right)$, a meromorphic section of $L_{s}$. To complete the analysis we have to display a generating section of $L_{s}$. Let $\breve{f}(z)=q_{1} b(z) u_{1} p$ where (recall $\left.g_{1}=q_{1} b_{1} p \in P\right) b(0) \in B$. Now $z^{x_{1}} q_{1}=$ $q_{1} z^{x_{1}}$ and $z^{x_{1}} b(z) z^{-x_{1}}$ is holomorphic at $z=0$. We therefore find that $\left(z^{x_{1}} q_{1} b(z) z^{-x_{1}} u_{1} p_{1}, 1\right)$ is a holomorphic section of $L_{s}$. Therefore, the contribution at $z=0$ to $\operatorname{deg}\left(L_{s}\right)-\operatorname{deg}\left(L_{f}\right)$ is $\gamma\left(x_{1}-u_{1}^{-1} x_{1}\right)$.

The calculation at $\infty$ is similar and we arrive at the equation in the statement.

Proof of Theorem 4. Now consider the map which takes a map $f: \mathbb{P}^{1} \rightarrow G / P$ to the map $s$ as above. We have seen that if we choose generic $g_{i}$ to compute the left-hand side, then the $s$ 's correspond to the right-hand side computed with respect to $g_{i}^{\prime}$. The $g_{i}^{\prime}$ depend only on the $g_{i}$ and the central elements chosen. So computed with respect to $g_{i}^{\prime}$ the right-hand side is a finite number and the codimension computation therefore gives us an inequality:

$$
\left\langle X_{u_{1}}, X_{u_{2}}, X_{u_{3}}\right\rangle_{Z} \leqslant\left\langle X_{w_{c_{1}} u_{1}}, X_{w_{c_{2}} u_{2}}, X_{u_{3}}\right\rangle_{Z^{\prime}}
$$

Now apply the reasoning again, this time with $c_{2}, c_{1}$, to get the other inequality.

Corollary 2. Let $Z \in H_{2}(G / P, \mathbb{Z}), u_{1}, \ldots, u_{s} \in W, c_{1}, \ldots, c_{s} \in \operatorname{center}(G), c_{1} c_{2} \ldots c_{s}=1$. Let $x_{k}=h_{c_{k}}$. Then,

$$
\left\langle X_{u_{1}}, \ldots, X_{u_{s}}\right\rangle_{Z}=\left\langle X_{w_{c_{1}} u_{1}}, \ldots, X_{w_{c_{s}} u_{s}}\right\rangle_{Z^{\prime}}
$$

where $Z^{\prime}$ as an element of $\operatorname{Hom}\left(W L_{P}, \mathbb{Z}\right)$ is given by

$$
Z^{\prime}(\gamma)=Z(\gamma)-\gamma\left(u_{1}^{-1} x_{1}\right)-\gamma\left(u_{2}^{-1} x_{2}\right)-\cdots-\gamma\left(u_{s}^{-1} x_{s}\right)
$$

Proof. Let us do the case $s=3$, the general case is similiar. We write down the transformation formulas (as in the theorem) for $c_{1}, c_{1}{ }^{-1}$, and then transform this on the second and third 'variables' by $c_{1} c_{2},\left(c_{1} c_{2}\right)^{-1}$; it is clear that $\left(c_{1} c_{2}\right)^{-1}=c_{3}$. We just have to verify that the formula for $Z^{\prime}$ is the one above. We leave this to the reader. 


\section{Reformulation}

Let $P$ be a standard parabolic and $\Sigma$ the set $\left\{\alpha \in \Delta \mid \mathfrak{g}_{-\alpha} \not \subset \mathfrak{p}\right\}$. It is clear that $\left\{\omega_{\sigma} \mid \sigma \in \Sigma\right\}$ is a basis of $W L_{P}$. Introduce variables $q_{\sigma}$ for $\sigma \in \Sigma$.

We need the following simple fact before we can describe the quantum cohomology of $G / P$.

Duals. The Poincare dual of the class $X_{w}$ is the class $X_{w_{0} w}$ where $w_{0}$ is the unique element in the Weyl group, so that

$$
B \cap w_{0} B w_{0}^{-1}=T
$$

Definition 3. Define the following.

1) $X_{u_{1}} \star X_{u_{2}}=\sum_{u \in W / W_{P}, Z \in \operatorname{Hom}\left(W L_{P}, \mathbb{Z}\right)}\left(\prod_{\sigma \in \Sigma} q_{\sigma}{ }^{Z\left(\omega_{\sigma}\right)}\right)\left\langle X_{u_{1}}, X_{u_{2}}, X_{u}\right\rangle_{Z} X_{w_{0} u}$.

2) $Q H(G / P)=H^{*}(G / P, \mathbb{C}) \otimes \mathbb{C}\left[q_{\sigma}: \sigma \in \Sigma\right]$ with the product given above.

3) For $c$ in center of $G$ with $w_{c}$ the associated Weyl group element, let $T_{c}: Q H(G / P) \rightarrow Q H(G / P)$ by

$$
T_{c}\left(X_{w}\right)=\left(\prod_{\sigma} q_{\sigma}{ }^{\omega_{\sigma}\left(w^{-1} h_{c}-h_{c}\right)}\right) X_{w_{c} w}
$$

with $h_{c}$ defined as in Definition 1.

Let us now try to compare $T_{c}\left(X_{u_{1}} \star X_{u_{2}}\right)$ to $T\left(X_{u_{1}}\right) \star X_{u_{2}}$.

\section{LEMMA 8.}

1) $T_{1}=$ multiplication by one.

2) $T_{c}(x \star y)=T_{c}(x) \star y$.

3) For $c_{1}, c_{2} \in \operatorname{center}(G)$, let $x_{1}=h_{c_{1}}, x_{2}=h_{c_{2}}$. Then,

$$
T_{c_{1}} T_{c_{2}}=\left(\prod_{\sigma} q_{\sigma}{ }^{\omega_{\sigma}\left(w_{c_{1}}^{-1} x_{2}-x_{2}\right)}\right) T_{c_{1} c_{2}}=\left(\prod_{\sigma} q_{\sigma}{ }^{\omega_{\sigma}\left(w_{c_{2}}^{-1} x_{1}-x_{1}\right)}\right) T_{c_{1} c_{2}}
$$

as operators.

\section{The $S L_{n}$ case}

Let us look at $\operatorname{Gr}(r, n)$. Here we have simple roots $L_{i}-L_{i+1}$ for $i=n-1, \ldots, 1$. For $\operatorname{Gr}(r, n), \Sigma$ from the previous section is $L_{r}-L_{r+1}$. The center is the cyclic group of order $n$ generated by the diagonal matrix $\Theta$ with entries $\zeta$, where $\zeta=e^{2 \pi i / n}$.

The element in $\bar{C}$ corresponding to $\Theta^{k}, k=1, \ldots, n-1$ is

$$
\left(\frac{k}{n}, \ldots, \frac{k}{n}, \frac{k}{n}-1, \frac{k}{n}-1\right),
$$

where there are $(n-k) k / n$ 's. The element of the Weyl group corresponding to $\Theta^{k}$ is just 'subtract $k$ modulo $n$, replacing zeros by $n$ ' in the standard representation of the Weyl group as a permutation group. Using these we can give a more explicit form of the transformation formulas.

Definition 4. Let $I=\left\{i_{1}<i_{2}<\cdots<i_{r}\right\}$. Let $F_{\bullet}$ be a complete flag in an n-dimensional vector space $E$. Now let $\Omega_{I}\left(F_{\bullet}\right)=\left\{L \in \operatorname{Gr}(r, E) \mid \operatorname{dim}\left(L \cap F_{i_{t}}\right) \geqslant t\right.$ for $\left.1 \leqslant t \leqslant r\right\}$. We denote the cohomology class of this subvariety by $\sigma(I)$. The codimension of this subvariety is the number of pairs $(j, i)$ with $j \notin I, i \in I$ and $j>i$. 


\section{P. Belkale}

The Gromov-Witten invariants in the Grassmannian case also have an interpretation in terms of vector bundles on $\mathbb{P}^{1}$. Let $\mathcal{V}=\mathcal{O}_{\mathbb{P}^{1}}^{n}$. We have a universal sequence of vector bundles on $\operatorname{Gr}(r, n)$

$$
0 \rightarrow \mathcal{S} \rightarrow \mathcal{V} \rightarrow \mathcal{Q} \rightarrow 0
$$

where $\mathcal{S}$ is the universal subbundle of rank $r$ and $\mathcal{Q}$ the quotient. It is now easy to verify that degree $d$ maps $\rho: \mathbb{P}^{1} \rightarrow \operatorname{Gr}(r, n)$ are in one-to-one correspondence with subbundles of rank $r$ and degree $-d$ of $\mathcal{V}$ by pulling back the universal sequence via the map $\rho$. Also, the image of point $p_{i}$ under this map is exactly the fiber of this subbundle at $p_{i}$. It is useful to fix an $n$-dimensional space $T$ and identify all the fibers of the bundle $\mathcal{V}$ with $T$. To obtain the other direction of this correspondence, note that subbundles $\mathcal{S}$ correspond to a family of $r$-dimensional subspaces of $T$ (over $\mathbb{P}^{1}$ ).

Now fix $s$ general flags on $T=\mathbb{C}^{n}: F_{p_{i}, \bullet}, i=1, \ldots, s$, as well as $s$ points $p_{1}, \ldots, p_{s}$ on $\mathbb{P}^{1}$. Let $I_{1}, \ldots, I_{s}$ be subsets of $\{1, \ldots, n\}$ of cardinality $r$ each, the Gromov-Witten number

$$
\left\langle\sigma\left(I_{1}\right), \ldots, \sigma\left(I_{s}\right)\right\rangle_{d}
$$

therefore counts the number ${ }^{1}$ of subbundles $\mathcal{S}$ of $\mathcal{V}$ of degree $-d$ and rank $r$ such that the fiber $\mathcal{S}_{p_{i}}$ as a subset of $T$ lies in the Schubert variety $\Omega_{I}\left(F_{p_{i}, \bullet}\right)$.

\subsection{The Transformation property}

Theorem 5. Let $I_{1}, \ldots, I_{s}$ be subsets of $\{1, \ldots, n\}$ of cardinality $r$ each. Let $n_{1}, \ldots, n_{s}$ be natural numbers summing to $n$. Define $J_{i}=I_{i}-n_{i} \bmod n$. That is, subtract $n_{i}$ from the numbers in $I_{i}$, reduce them $\bmod n$ and replace all zeros by $n$. Define $d_{i}$ as the number of elements in $I_{i}$ which are less than or equal to $n_{i}$, then

$$
\left\langle\sigma\left(I_{1}\right), \ldots, \sigma\left(I_{s}\right)\right\rangle_{d}=\left\langle\sigma\left(J_{1}\right), \ldots, \sigma\left(J_{s}\right)\right\rangle_{d+r-\sum d_{i}} .
$$

Remark. This property has been noted and proved in [AW98] as a consequence of the Schubert calculus established in [Ber97]. The proof here is geometric and independent of [Ber97]. It is essentially the same proof as that of the transformation formulas of the previous section.

Proof. First we verify that the codimension conditions on both sides are the same. That is

$$
\sum_{i=1}^{s} \operatorname{codim}\left(\sigma\left(I_{i}\right)\right)=n d+r(n-r)
$$

is same as the condition

$$
\sum_{i=1}^{s} \operatorname{codim}\left(\sigma\left(J_{i}\right)\right)=n\left(d+r-\sum d_{i}\right)+r(n-r) .
$$

This follows easily from the observation

$$
\operatorname{codim}\left(\sigma\left(J_{i}\right)\right)=\operatorname{codim}\left(\sigma\left(I_{i}\right)\right)+\left(n_{i}-d_{i}\right) r-(n-r) d_{i} .
$$

Now fix $s$ general flags on $V=\mathbb{C}^{n}: F_{p_{i}, \bullet}, i=1, \ldots, s$, as well as $s$ points $p_{1}, \ldots, p_{s}$ on $\mathbb{P}^{1}$. Then $\left\langle\sigma\left(I_{1}\right), \ldots, \sigma\left(I_{s}\right)\right\rangle_{d}$ is the number of subbundles (zero if there is an infinite family) of subbundles $\mathcal{E}$ of $\mathcal{V}=V \bigotimes_{\mathbb{C}} \mathcal{O}$ of degree $-d$ and rank $r$ such that the fiber $\mathcal{E}_{p_{i}}$ as a subset of $\mathbb{C}^{n}$ lies in the Schubert variety $\Omega_{I_{i}}\left(F_{p_{i}, \bullet}\right)$. Let $V_{i}=F_{p_{i}}^{n_{i}}$. From the genericity of the flags, we have an equality

$$
\bigoplus V_{i} \rightarrow \mathbb{C}^{n}
$$

\footnotetext{
${ }^{1}$ It is defined to be zero if there is an infinite family of such subbundles.
} 
We define a new bundle on $\mathbb{P}^{1}$ as follows ( $q$ is a new point on $\mathbb{P}^{1}$ ).

$$
\mathcal{V}^{\prime}=\bigoplus\left(V_{i} \bigotimes \mathcal{O}\left(p_{i}-q\right)\right)
$$

Note that we are given an isomorphism $\mathcal{V} \rightarrow \mathcal{V}^{\prime}$ over the open set $U=\mathbb{P}^{1}-\left\{p_{1}, \ldots, p_{s}, q\right\}$ and also that $\mathcal{V}^{\prime}$ is isomorphic to $\mathcal{O}^{n}$. From the theorem below we know that we have a one-to-one correspondence between subbundles $\mathcal{E}$ of $\mathcal{V}$ and subbundles $\mathcal{E}^{\prime}$ of $\mathcal{V}^{\prime}$. We also have induced flags $F_{p_{i}, \bullet}^{\prime}$ on the fibers of $\mathcal{V}^{\prime}$ at the points $p_{i}$, so that if fiber $\mathcal{E}_{p_{i}}$ is in the Schubert variety $\Omega_{I_{i}}\left(F_{p_{i}, \bullet}\right)$, then the fiber $\mathcal{E}_{p_{i}}^{\prime}$ is in the Schubert variety $\Omega_{J_{i}}\left(F_{p_{i}, \bullet}^{\prime}\right.$, . The theorem below also tells us that in this correspondence, the degree of $\mathcal{E}^{\prime}$ is equal to the degree of $\mathcal{E}-r+\sum d_{i}$.

This finishes the proof, but we have to deal with genericity questions. A more refined approach can directly show that the induced flags on $\mathcal{V}^{\prime}$ are generic too. However, we wish to avoid this line of argument here. Instead we note that this argument proves (with the codimension computation) that $\left\langle\sigma\left(I_{1}\right), \ldots, \sigma\left(I_{s}\right)\right\rangle \leqslant\left\langle\sigma\left(J_{1}\right), \ldots, \sigma\left(J_{s}\right)\right\rangle_{d+r-\sum d_{i}}$.

We could then reverse this construction to get the other inequality. This proves that there are no intersections at 'infinity' and also transversality without invoking the theory of Quot schemes.

Theorem 6 (Local theory). Let $C$ be a smooth curve $p$ a point on it and $t$ a uniformising parameter at $p$. Let $\mathcal{V}$ be a vector bundle on $C$ and $V=$ fiber $_{p}$. Also, suppose that we are given a complete flag on $V: V_{1} \subset V_{2} \subset \cdots \subset V_{n}=V$. Define $\mathcal{V}_{k}^{\prime}=\{$ meromorphic sections $s$ of $\mathcal{V}$ which are holomorphic sections of $\mathcal{V}$ outside of $p$ and such that ts extends to give a section of $\mathcal{V}$ near $p$ with a fiber at $p$ in $\left.V_{k}\right\}$.

1) $\mathcal{V} \subset \mathcal{V}_{k}^{\prime}$ with quotient supported at $p$ of dimension $k$.

2) There is a one-to-one correspondence between subbundles $\mathcal{E}$ of $\mathcal{V}$ and subbundles $\mathcal{E}_{k}^{\prime}$ of $\mathcal{V}_{k}^{\prime}$. In this correspondence the quotient $\mathcal{E}_{k}^{\prime} / \mathcal{E}$ is supported at $p$ and has $\operatorname{dimension} \operatorname{dim}\left(\mathcal{E}_{p} \cap V_{k}\right)$.

3) We have a sequence of inclusions

$$
t\left(\mathcal{V}_{k}^{\prime}\right) \subset t\left(\mathcal{V}_{k+1}^{\prime}\right) \subset \cdots \subset \mathcal{V} \subset \mathcal{V}_{1}^{\prime} \cdots \subset \mathcal{V}_{k}^{\prime}
$$

which gives a complete flag on the fiber $\left(\mathcal{V}_{k}^{\prime}\right)_{p}$.

4) In the correspondence on the subbundles, if fiber $\mathcal{E}_{p}$ is in the Schubert variety $\Omega_{I}\left(F_{p, \bullet}\right)$, then the fiber $\left(\mathcal{E}_{k}^{\prime}\right)_{p}$ is in the Schubert variety $\Omega_{I-k}\left(F_{p, \bullet}^{\prime}\right)$.

The proofs are all fairly obvious and can be found in the appendix to [Bel01].

\subsection{Quantum Schubert calculus}

The objective of this section is to show how Pieri's formula is an easy consequence of the relations of the previous section. Namely for the intersections in the Pieri formula, the relations reduce to the $d=0$ case and are classically known by induction.

As in the classical approach of Hodge-Pedoe (see [GH78]), we can then prove the Giambelli formula from Pieri. In [Ber97], Pieri is deduced from Giambelli.

Definition 5. Define a map $T: Q H(\operatorname{Gr}(r, n)) \rightarrow Q H(\operatorname{Gr}(r, n))$ by the rule $T(\sigma(I))=q^{d_{1}}(\sigma(I-1))$, where $d_{1}$ is the number of elements in $I$ which are less than or equal to one. Note that if $k \leqslant n$, $T^{k}(\sigma(I))=q^{d_{k}} \sigma(I-k)$, where $d_{k}$ is the number of elements in $I$ which are less than or equal to $k$. This $T$ is essentially $T_{\Theta}$ of the previous section.

THEOREM 7 (Reformulation of transformation property). The transformation property is equivalent to the property

$$
T(\sigma(I) \star \sigma(J))=T(\sigma(I)) \star \sigma(J) .
$$




\section{P. Belkale}

The proof of Pieri is pure algebra beyond this point. We first note the following.

Lemma 9. Suppose $\sigma(I)$ has codimension less than or equal to $n-1$ and that $\sigma(K)$ appears in $\sigma(I) \star \sigma(J)$ with a $q$ coefficient greater than or equal to one, then there exists $k$ so that $\sigma(K-k)$ appears in $\sigma(I) \star \sigma(J-k)$ with $q$ degree 0 .

Proof. Suppose not, choose $k$ so that the $q$ degree is minimized and equals $d$. Let $J^{\prime}=J-k$ and $K^{\prime}=K-k$. Since we cannot minimize the $q$ degree further, application of the operation $T^{l}$ tells us that if $d_{l}$ is the number of elements in $J^{\prime}$ which are less than or equal to $l$, and $c_{l}$ is the similar number for $K^{\prime}$, then $d_{l} \leqslant c_{l}$. This clearly implies that $\operatorname{codim}\left(J^{\prime}\right) \leqslant \operatorname{codim}\left(K^{\prime}\right)$. However, we also have

$$
\operatorname{codim}(I)+\operatorname{codim}\left(J^{\prime}\right)=n d+\operatorname{codim}\left(K^{\prime}\right) .
$$

This yields a contradiction immediately if $d \geqslant 1$.

Pieri's formula is usually written in cohomological notation. For this, we make the following definition.

Definition 6. If $I=\left\{i_{1}<\cdots<i_{r}\right\}$ is a subset of $\{1, \ldots n\}$, then define $a(I, k)=n-r+k-i_{k}$, for $k=1, \ldots, r$.

Definition 7 (Special Schubert cells). If $a \leqslant n-r$ define $\sigma_{a}=\sigma\left(I_{a}\right)$ where

$$
I_{a}=\{n-r+1-a, n-r+2, \ldots, n-r\} .
$$

THEOREM 8.

$$
\sigma_{a} \star \sigma(I)=\sum_{K} \sigma(K)+q \sum_{L} \sigma(L),
$$

where the $K$ sum is over all $K$ satisfying

$$
n-r \geqslant a(K, 1) \geqslant a(I, 1) \geqslant a(K, 2) \geqslant \cdots \geqslant a(K, r) \geqslant a(I, r)
$$

and

$$
\operatorname{codim}(I)+a=\operatorname{codim}(K)
$$

and the $L$ sum is over all $L$ satisfying

$$
a(I, 1)-1 \geqslant a(L, 1) \geqslant a(I, 2)-1 \geqslant \cdots \geqslant a(I, r)-1 \geqslant a(L, r) \geqslant 0
$$

and

$$
\operatorname{codim}(I)+a=\operatorname{codim}(L)+n .
$$

Note that there are no $L$ terms if $a(I, r)=0$.

Proof. The statement about the $K$ terms is classical [GH78]. Let us first show that there are no terms with $q^{2}$ and higher. From the previous lemma there is then a $k$ so that if $I^{\prime}=I+k$ and $L^{\prime}=L+k$, we have:

- $\sigma\left(L^{\prime}\right)$ appears with $q$ degree 0 in $\sigma_{a} \star \sigma\left(I^{\prime}\right)$;

- $d_{k} \leqslant c_{k}-2$, where $d_{k}$ is the number of elements in $I^{\prime} \leqslant k$ and $c_{k}$ is the number of elements in $L^{\prime} \leqslant k$.

Let $d_{k}=j$. Then $a\left(L^{\prime}, j+2\right) \leqslant a\left(I^{\prime}, j+1\right)$ tells us that if $L^{\prime}=\left\{l_{1}^{\prime} \leqslant \cdots \leqslant l_{r}^{\prime}\right\}$ and $I^{\prime}=\left\{i_{1}^{\prime} \leqslant\right.$ $\left.\cdots \leqslant i_{r}^{\prime}\right\}$, then

$$
n-r+(j+1)-l_{j+2}^{\prime} \leqslant n-r+j-i_{j+1}^{\prime},
$$

or what is the same $i_{j+1}^{\prime}+1 \leqslant l_{j+2}^{\prime}$, hence $l_{j+2}^{\prime} \geqslant i_{j+1}^{\prime}+1$. However, $i_{j+1}^{\prime}>k$, therefore $l_{j+2}^{\prime}>k+1$ which is in direct contradiction to $d_{k} \leqslant c_{k}-2$. 
We now deal with the $q^{1}$ terms. We find $L^{\prime}$ and $I^{\prime}$ as before so that $d_{k}=c_{k}-1$. Let $d_{k}=j, c_{k}=$ $j+1$. Then contemplation of $I=I^{\prime}-k$ and $J=J^{\prime}-k$ gives the result. For example, let us only verify $a(I, r)-1 \geqslant a(L, r)$. It is clear that $l_{r}=l_{j+1}^{\prime}-k+n$ and $i_{r}=i_{j}^{\prime}-k+n$. So we need to verify that

$$
n-r+r-l_{r} \leqslant n-r+r-i_{r}-1
$$

or that,

$$
i_{r}+1 \leqslant l_{r}
$$

or that,

which is just

$$
i_{j}^{\prime}+1 \leqslant l_{j+1}^{\prime},
$$

$$
a\left(I^{\prime}, j\right) \geqslant a\left(L^{\prime}, j+1\right),
$$

but this is already known classically.

The tediousness of the above proof is made up by the simplicity of the proof of Giambelli formula. However, before that we need a definition.

Definition 8 . Let $\breve{a}=a_{1} \geqslant \cdots \geqslant a_{r}$ be given, then define $I(\breve{a})$ by $i_{l}=n-r+l-a_{l}$. If this is not a subset of $\{1, \ldots, n\}$ define $\sigma(I(\breve{a}))$ to be zero. Also denote $\sigma(I(\breve{a}))$ by $\sigma_{a_{1}, \ldots, a_{r}}$ and ignore zeros in the subscript if they appear.

Theorem 9.

$$
(-1)^{d} \sigma_{a_{1}, \ldots a_{d}}=\sum_{j=1}^{d}(-1)^{j} \sigma_{a_{1}, \ldots, a_{j-1}, a_{j+1}-1, \ldots, a_{d}-1} \star \sigma_{a_{j}+d-j} .
$$

Proof. Note that on the left-hand side the length of the string $\breve{a^{\prime}}=\left\{a_{1}, \ldots, a_{j-1}, a_{j+1}-1, \ldots, a_{d}-1\right\}$ is less than $r$. So $a_{r}^{\prime}=0$. Hence no $q^{1}$ terms are produced via Pieri, and the formula in $q$ degree 0 is known classically [GH78, p. 205]. Hence there is really nothing (new) to prove. Iteration of this gives the Giambelli formula as in [GH78].

Fulton and Woodward [FW] have proved a theorem on the smallest power of $q$ in the quantum product of Schubert subvarieties in the case of $G / P, P$ maximal parabolic. We prove the Grassmann case of their theorem in a slightly strengthened form.

Theorem 10 (Fulton-Woodward). The smallest power of $q$ appearing in $\sigma(I) \star \sigma(J)$ is the number $d=\max \left\{d_{i}+d_{j}^{\prime}-r \mid i+j=n\right\}$, where $d_{i}$ is the number of elements in $I$ which are less than or equal to $i, d_{j}^{\prime}$ is the number of elements in $J$ which are less than or equal to $j$. Moreover, if the max is achieved for $i, j: i+j=n$, then

$$
\sigma(I) \star \sigma(J)=q^{d}(\sigma(I-i) \cup \sigma(J-j))+\text { higher-order terms, }
$$

where the cup product $\cup$ on the right-hand side is the cup product in the usual cohomology.

Remark. The 'strengthened' part refers to identification of the lowest order terms which is curiously a product in the ordinary cohomology. This may not be true for all $G / P$ 's. Also, the associativity of quantum cohomology has not been used so far!

Proof. We know $T^{n}$ is equal to multiplication by $q^{r}$, therefore if $i+j=n$,

$$
\begin{aligned}
q^{r}(\sigma(I) \star \sigma(J)) & =T^{n}(\sigma(I) \star \sigma(J)) \\
& =T^{i}(\sigma(I)) \star T^{j}(\sigma(J)) \\
& =q^{d_{i}+d_{j}^{\prime}} \sigma(I-i) \star \sigma(J-j) .
\end{aligned}
$$


Therefore, if $i+j=n$,

$$
\sigma(I) \star \sigma(J)=q^{d_{i}+d_{j}^{\prime}-r} \sigma(I-i) \star \sigma(J-j) .
$$

Now choose $i, j\left(\right.$ with sum $=n$ ) so as to maximize $d_{i}+d_{j}^{\prime}-r$. Let $I^{\prime}=I-i$ and $J^{\prime}=J-j$. Define $c_{k}$ as the number of elements in $I^{\prime}$ which are less than or equal to $k$ and $c_{k}^{\prime}$ as the number of elements in $J^{\prime}$ which are less than or equal to $k$.

We clearly have $c_{k}+c_{n-k}^{\prime} \leqslant r$, for any $k$. This tells us that the dual of $\sigma\left(I^{\prime}\right)$ is contained in $\sigma\left(J^{\prime}\right)$, for a choice of flags. Hence, by Kleiman's Bertini theorem, we have $\sigma\left(I^{\prime}\right) \cap \sigma\left(J^{\prime}\right) \neq 0$.

\section{ACKNOWLEDGEMENTS}

I thank A. Bertram, P. Brosnan, A. Buch, W. Fulton, M. Kapovich, M. V. Nori and C. Woodward for useful comments.

\section{REFERENCES}

AW98 S. Agnihotri and C. Woodward, Eigenvalues of products of unitary matrices and quantum Schubert calculus, Math. Res. Lett. 5 (1998), 817-836.

Bel01 P. Belkale, Local systems on $\mathbb{P}^{1}-S$ for $S$ a finite set, Compositio Math. 129 (2001) 67-86.

Ber97 A. Bertram, Quantum Schubert calculus, Adv. Math. 128 (1997), 289-305.

Bou02 N. Bourbaki, Lie groups and Lie Algebras, ch. 4-6, Elements of Mathematics (Berlin) (SpringerVerlag, Berlin, 2002).

Buc03 A. Buch, Quantum cohomology of Grassmannians, Compositio Math. 137 (2003), 227-235.

FW W. Fulton and C. Woodward, On the quantum product of Schubert classes, J. Algebraic Geom., to appear.

GH78 P. Griffiths and J. Harris, Principles of algebraic geometry (Wiley Interscience, 1978).

Kim99 B. Kim, Quantum cohomology of Flag manifolds $G / B$ and quantum Toda lattice, Ann. Math. 149, (1999), 129-148.

KT03 A. Kresch and H. Tamvakis, Quantum cohomology of the Lagrangian Grassmannian, J. Algebraic Geom. 12 (2003), 777-810.

KT04 A. Kresch and H. Tamvakis, Quantum cohomology of orthogonal Grassmannians, Compositio Math. 140 (2004), 482-500.

Mar02 A-L. Mare, Polynomial representatives of Schubert classes in $Q H^{*}(G / B)$, Preprint (2002) (math.CO/ 0205309).

Pos00 A. Postnikov, Symmetries of Gromov-Witten invariants, Preprint (2000) (math.CO/0009174).

TW03 C. Teleman and C. Woodward, Parabolic bundles, products of conjugacy classes and quantum cohomology, Ann. Inst. Fourier, Tome 53, fasc. 3 (2003), 713-748.

Woo02 C. Woodward, On D. Peterson's comparison formula for Gromov-Witten invariants, Preprint (2002) (math.AG/0206073).

Prakash Belkale belkale@email.unc.edu

Department of Mathematics, UNC-Chapel Hill, CB \#3250, Phillips Hall, Chapel Hill, NC 27599, USA 